\title{
Erratum: Wald Entropy for Ghost-Free, Infinite Derivative Theories of Gravity [Phys. Rev. Lett. 114, 201101 (2015)]
}

\author{
Aindriú Conroy, Anupam Mazumdar, and Ali Teimouri
}

(Received 23 May 2017; revised manuscript received 16 October 2017; published 19 January 2018)

DOI: 10.1103/PhysRevLett.120.039901

We wish to make the following corrections to the original Letter. The corrections are as follow: (1) The abstract shall be corrected as follows: "In this Letter, we demonstrate that the Wald entropy for any spherically symmetric black hole within an infinite derivative theory of gravity that is quadratic in curvature is determined, in the linearized regime solely by the area law. (By linearized regime, we refer to a perturbation of the metric tensor around Minkowski space according to the prescription $g_{\mu \nu}=\eta_{\mu \nu}+h_{\mu \nu}$, up to linear order in $h$. That is, we need not consider terms of higher order than $h^{2}$ in the gravitational action. The resulting calculation serves as a first order approximation of the gravitational entropy.) Thus, the infrared behavior of gravity is captured by the Einstein-Hilbert term, provided that the massless graviton remains the only propagating degree of freedom in the spacetime. In the nonlinear regime, however, the entropy will be modified by appropriate corrections." (2) In the nonlinear regime, the gravitational entropy for infinite derivative gravity (IDG) is not given by the area law as described by Eq. (10) and Eq. (17) of the Letter. As such, these equations should be disregarded. We would like to thank Yun Soo Myung for helpful discussions on the matter [1]. Additionally, it is preferable to express a general expression for gravitational entropy given by Eq. (9) in the following form:

$$
S_{W}=\frac{A_{H}}{4 G}\left\{1+\alpha\left[2 \mathcal{F}_{1}\left(\square_{M}\right)+\mathcal{F}_{2}\left(\square_{M}\right)+2 \mathcal{F}_{3}\left(\square_{M}\right)\right] g^{a b} R_{a b}+2 \alpha\left[\mathcal{F}_{1}\left(\square_{M}\right) g^{i j} R_{i j}-\mathcal{F}_{3}\left(\square_{M}\right) g^{a b} g^{i j} R_{i a j b}\right]\right\} .
$$

Here, $(a, b)$ correspond to $(r, t)$ directions and $(i, j)$ correspond to the spherical coordinates $(\theta, \phi)$. This result was also obtained later in Ref. [2]. We also note that $\square_{M} \equiv \square / M^{2}$, where $M$ denotes mass, this is to be dimensionally consistent. (3) In the linearized regime, the gravitational entropy does indeed reduce to the area law as stated in the Letter. However, we wish to amend the argument in this case. (i) In this case, the metric given in Eq. (18) should we written in its spherically symmetric form,

$$
d s^{2}=-[1+2 \Phi(r)] d t^{2}+[1-2 \Psi(r)] d r^{2}+r^{2} d \Omega^{2},
$$

where $d \Omega^{2}=d \theta^{2}+\sin ^{2}(\theta) d \phi^{2}$. (ii) By employing the ghost-free constraint $\left[2 \mathcal{F}_{1}\left(\square_{M}\right)+\mathcal{F}_{2}\left(\square_{M}\right)+2 \mathcal{F}_{3}\left(\square_{M}\right)=0\right]$, we find the IDG entropy for the above metric to be

$$
S_{W}=\frac{A_{H}}{4 G}\left[1+2 \Psi(r)-2 \Phi(r)-4 \alpha \mathcal{F}_{3}\left(\square_{M}\right)\left(\frac{\Psi^{\prime}(r)-\Phi^{\prime}(r)}{r}\right)\right] .
$$

This equation should take the place of Eq. (19). We further note that we have used the spherical form of the D'Alembertian operator:

$$
\square u=\frac{1}{r^{2}} \partial_{r}\left(r^{2} \partial_{r} u\right)+\frac{1}{r^{2} \sin \theta} \partial_{\theta}\left(\sin \theta \partial_{\theta} u\right)+\frac{1}{r^{2} \sin ^{2} \theta} \partial_{\varphi}^{2} u-\frac{1}{c^{2}} \partial_{t}^{2} u .
$$

It can be seen from Eq. (3) that for $\Psi(r)=\Phi(r)$ the entropy would be given solely by the area law. Note that in order to make sure that the graviton remains massless all the way from infrared to ultraviolet regime, and recovers the success of GR in the infrared, we imposed the constraint $2 \mathcal{F}_{1}\left(\square_{M}\right)+\mathcal{F}_{2}\left(\square_{M}\right)+2 \mathcal{F}_{3}\left(\square_{M}\right)=0$.

We would like to clarify that the source of the mistake in the Letter was a computational one. The mistake was related to decomposing the curvature appropriately [see Eq. (1)]. The correct entropy later calculated later on Ref. [2] so it was necessary to correct the earlier work. 
[1] Y. S. Myung, Entropy of a black hole in infinite-derivative gravity, Phys. Rev. D 95, 106003 (2017).

[2] A. Conroy, A. Mazumdar, S. Talaganis, and A. Teimouri, Nonlocal gravity in D dimensions: Propagators, entropy, and a bouncing cosmology, Phys. Rev. D 92, 124051 (2015). 